\title{
Género y empoderamiento comunitario en un contexto de posconflicto: el caso de Vergara, Cundinamarca (Colombia)*
}

\author{
Gender and Community Empowerment in a Post-Conflict Context: \\ The Case of Vergara, Cundinamarca (Colombia) \\ Gênero e empoderamento comunitário em um contexto de pós-conflito: \\ o caso de Vergara, Cundinamarca (Colômbia)
}

\author{
LEONARDO GÜIZA SUÁREZ ${ }^{\text {** }}$ \\ CRISTHIAN DAVID RODRÍGUEZ-BARAJAS ${ }^{* * *}$ \\ Blanca Oliva Ríos Osorio ${ }^{* *+*}$ \\ SARA Sofía Moreno Gallo ${ }^{* * * * x}$
}

FECHA DE RECEPCIÓN: 11 DE NOVIEMBRE DE 2015. FECHA DE ACEPTACIÓN: 13 DE MARZO DE 2016

DOI: DX.DOI.ORG/10.12804/ESJ 18.02.2016.04

Para citar este artículo: Güiza, L., Rodríguez-Barajas, C., Ríos, B. y Moreno, S. (2016). Género y empoderamiento comunitario en un contexto de posconflicto: el caso de Vergara, Cundinamarca (Colombia). Estudios Socio-Jurídicos, 18(2), $117-146$. Doi: dx.doi.org/10.12804/esj18.02.2016.04

* El presente artículo se desarrolló en el marco del Convenio de Asociación No. 1257 de 2014 suscrito entre la Corporación Autónoma Regional de Cundinamarca (CAR) y el Colegio Mayor de Nuestra Señora del Rosario, con el fin de aunar esfuerzos para adelantar la segunda fase del proyecto "Hacia un observatorio de agendas interinstitucionales y conflictos socio-ambientales con enfoque de derechos humanos en el territorio de la Corporación".

** Magíster en Derecho Ambiental Industrial y magíster en Derechos Humanos. Profesor de carrera académica de la Facultad de Jurisprudencia de la Universidad del Rosario. Correo electrónico: leonardo.guiza@urosario.edu.co

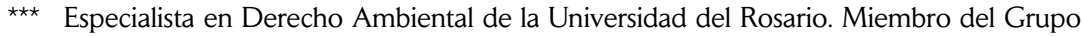
de investigación en Derechos Humanos y Medio Ambiente, de la Facultad de Jurisprudencia de la Universidad del Rosario. Correo electrónico: cristian_rb89@hotmail.com, rodriguezb.cristhian@ urosario.edu.co

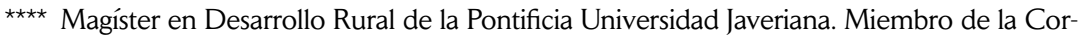
poración Autónoma Regional de Cundinamarca (CAR). Correo electrónico: olivarioso@yahoo.com

${ }_{\star \star \star \star \star \star}$ Abogada. Joven investigadora e innovadora de Colciencias en el Grupo de investigación en Derechos Humanos y Medio Ambiente de la Universidad del Rosario. Correo electrónico: sarasofia. morenog@gmail.com 


\section{RESUMEN}

La población rural femenina ha sido vulnerable a los impactos de la desigualdad social y el conflicto armado. Por esta razón, ante condiciones de discriminación, pobreza y violencia, algunas mujeres rurales han encontrado alternativas de cohesión comunitaria a partir de la conservación ambiental, las cuales se han vuelto una forma de mitigar y hacerle frente a esas dificultades. Este artículo muestra los resultados de un estudio de caso llevado a cabo en Vergara, Cundinamarca (Colombia). El objetivo es analizar las acciones de participación comunitaria implementadas por los miembros de la vereda Llano Grande, con el propósito de identificar las potencialidades, dificultades y lecciones aprendidas en torno a la gobernanza ambiental y al empoderamiento comunitario. Se hizo uso de entrevistas a profundidad, encuestas semiestructuradas, observación participante y grupos focales, mediante un modelo de Investigación-Acción Participativa (IAP), para definir estrategias de fortalecimiento local, igualdad de género y gobernanza ambiental en un contexto rural de posconflicto. Se sostiene que la participación comunitaria ambiental ha sido un instrumento para el empoderamiento de las mujeres rurales en dicho contexto $\mathrm{y}$, al mismo tiempo, contribuye positivamente a la consolidación de buenas prácticas de gobernanza ambiental en una perspectiva de género.

Palabras clave: género, posconflicto, mujeres rurales, conservación ambiental, Vergara.

\section{ABSTRACT}

The female rural population has been particularly vulnerable to the impacts of social inequality and armed conflict. For this reason, and against conditions of discrimination, poverty, and violence, some rural women have found alternatives of community cohesion from environmental conservation, which have become a way of mitigating and facing those difficulties. This paper shows the results of a case study carried out in Vergara, Cundinamarca (Colombia). Our objective is to analyze the actions of community participation implemented by the members of the vereda Llano Grande, in order to identify the potentiality, difficulties, and lessons learned around environmental governance and community empowerment. Use was made of in-depth interviews, semi-structured interviews, participant observation, and focus groups, through a model of Participatory Action Research (PAR), to define strategies of local strengthening, gender equality, and environmental governance in a post-conflict rural context. We will argue that the environmental community participation has been an instrument for rural women's empowerment in a post-conflict context while making a positive contribution to the consolidation of best practices of environmental governance in a gender perspective.

Keywords: gender, post-conflict, rural women, environmental conservation, Vergara.

\section{RESUMO}

A população rural feminina tem sido vulnerável aos impactos da desigualdade social e o conflito armado. Por esta razão, ante condições de discriminação, pobreza e violência, algumas mulheres rurais têm encontrado alternativas de coesão comunitária a partir da conservação ambiental, as quais se têm tornado uma forma de mitigar e fazer-lhe frente a estas dificuldades. Este artigo mostra os resultados de um estudo de caso levado a cabo em Vergara, Cundinamarca (Colômbia). O objetivo é analisar as ações de participação comunitária implementadas pelos membros da Vereda Llano Grande, com o propósito de identificar as potencialidades, dificuldades e lições aprendidas em torno à governança ambiental e ao empoderamento comunitário. Se fez uso de entrevistas a profundidade, questionários semiestruturados, observação participante e grupos focais, mediante um modelo de Investigação-Ação Participativa (IAP), para definir estratégias de fortalecimento local, igualdade de gênero e governança 
ambiental em um contexto rural de pós-conflito. Sustenta-se que a participação comunitária ambiental tem sido um instrumento para o empoderamento das mulheres rurais em dito contexto e, ao mesmo tempo, contribui positivamente à consolidação de boas práticas de governança ambiental em uma perspectiva de gênero.

Palavras- chave: gênero, pós-conflito, mulheres rurais, conservação ambiental, Vergara.

\section{Introducción}

El sector rural en América Latina es un escenario complejo en el que confluyen diferentes problemáticas como la depredación ambiental, las condiciones de pobreza, la pérdida de soberanía alimentaria, la globalización de la agricultura, la migración y el inapropiado uso y manejo de los recursos naturales (Almeyra, 2012; Ramírez-Miranda, 2014).

En Colombia, cerca de $47 \%$ de los habitantes sobreviven por debajo de la línea de pobreza, es decir, en el hogar, los ingresos promedio mensuales de sus integrantes son menores a COP $\$ 187.079$, lo que corresponde a cerca de 56 Euros. Esta situación se agudiza en el sector rural, donde dos terceras partes de la población viven bajo la línea de pobreza, las condiciones de vulnerabilidad y desigualdad en su población son notorias y el conflicto armado y la violencia agravan aún más la situación (Parra-Peña, Isidro, Ordoñez, Acosta, Perry, 2010).

Asimismo, las mujeres rurales han sido objeto de una histórica discriminación y desigualdad global, pues las estructuras patriarcales han sido propias de las relaciones sociales en el ámbito campesino. Por ejemplo, las labores de atención al hogar y a la familia, culturalmente asignadas al género femenino, han ubicado a la mujer en una posición de vulnerabilidad y escasez de oportunidades de desarrollo y formación (Álvarez, Díaz y Saiz, 2012).

Tanto las condiciones de marginación del sector rural como la posición de desigualdad del género femenino hacen necesario pensar en propuestas relacionadas con el empoderamiento de estas mujeres, a partir de la promoción de su participación efectiva en el avance de las comunidades de las que forman parte. De esta manera, se les otorga la posibilidad de intervenir en las situaciones y problemáticas que les afectan, apropiarse de su 
territorio, interactuar con los miembros de la comunidad y generar sinergias para lograr un desarrollo sostenible (Buendía-Martínez y Carrasco, 2013).

Esto responde a la creciente necesidad de inclusión de los temas de género en la agenda pública y de reconocimiento de las mujeres como actores sociales protagónicos, agentes de cambio y evolución social. Según el Consejo Nacional de Planeación, Económica y Social (Departamento Nacional de Planeación, 2013), el anterior es un tema de alta prioridad para el Estado colombiano, en el sentido de que esta inclusión y este reconocimiento promueven la garantía del acceso, el ejercicio y el goce de los derechos de las mujeres y contribuyen a superar las condiciones de desigualdad y las formas de discriminación y subordinación que persisten en nuestra sociedad.

El municipio de Vergara, ubicado en el departamento de Cundinamarca, en la región central de Colombia, fue durante mucho tiempo un escenario de disputa armada por parte de grupos militares, paramilitares y guerrilleros. En la década del noventa se intensificó el accionar de estos grupos, lo que afectó en repetidas ocasiones a la población local de forma directa (Vicepresidencia de la República de Colombia, 2001). Esto, sumado a las dificultades socioeconómicas y de desigualdad de género, causó una presión negativa sobre la población local y la vulneración progresiva de sus derechos humanos (Pérez, 2005).

Según manifestaron algunos de los pobladores más antiguos de Vergara en el desarrollo de los grupos focales, en la segunda mitad del siglo XX, surgieron constantes conflictos entre los pobladores del municipio por razones personales o familiares, que comenzaron a traducirse en el uso de la violencia para tratar de resolverlos. Más adelante entraron en la escena grupos guerrilleros y paramilitares, los cuales desencadenaron múltiples enfrentamientos bélicos en los que la población civil se veía involucrada. Incluso hubo asesinatos selectivos y masacres que dejaron numerosas víctimas.

De acuerdo con algunos pobladores de Vergara, desde el año 2005 los grupos armados dejaron el territorio y, junto con ellos, la guerra se ausentó de la vida local. Desde ese entonces, la comunidad rural de Vergara, en especial las mujeres víctimas de la violencia y las desigualdades socioeconómicas, han participado en iniciativas propias de un proceso de superación del conflicto armado y adaptación al contexto de posconflicto, 
lo que constituye un entorno fructífero para avanzar en la investigación de temas de género y desarrollo rural.

En el marco de un proyecto financiado por la Corporación Autónoma Regional de Cundinamarca (CAR, autoridad ambiental de la región), se observó la necesidad de avanzar en la investigación y aplicación de una agenda interinstitucional ambiental (AIA). Esta última es un instrumento de gestión ambiental rural participativa, construido sobre bases de inclusión, empoderamiento comunitario, equidad de género y cooperación de varios actores, con el fin de conocer y promover las valiosas iniciativas comunitarias que han surgido en este territorio.

En este artículo se presentan los resultados más importantes alcanzados en esta primera fase de diagnóstico y fortalecimiento comunitario. Así pues, el objetivo de este documento es analizar las acciones fundadas en la participación comunitaria con enfoque de género adelantadas por los miembros de la vereda Llano Grande, con el propósito de identificar las potencialidades, dificultades y lecciones aprendidas en torno a la gobernanza ambiental y al empoderamiento comunitario. La tesis defendida es que la participación comunitaria ambiental puede ser $-\mathrm{y}$, de hecho, ha sido- un instrumento de empoderamiento de las mujeres rurales en un contexto de posconflicto.

Se eligió como caso de estudio la experiencia de la Asociación de Productoras Líderes de Vergara (Asoproliv), con el fin de hacer un diagnóstico participativo para determinar de qué manera han interactuado elementos como la transformación de las relaciones de género, la renovación del sector rural y la gobernanza ambiental en su propio proceso de adaptación, organización y participación en el contexto de posconflicto que allí se presentó. Además, al escoger esta organización, se pudieron establecer de manera participativa las medidas y los lineamientos necesarios para promover el proceso de empoderamiento de esta asociación de mujeres y avanzar en su consolidación como un actor local protagónico en la gestión ambiental participativa y en el progreso rural del municipio.

Mediante un trabajo de campo en la vereda Llano Grande, zona donde habitan las integrantes de Asoproliv, se hizo un diagnóstico participativo a partir de observación participante, entrevistas semiestructuradas y grupos focales. Se obtuvo un panorama integral de las percepciones, experiencias y expectativas frente al empoderamiento comunitario con enfoque de género 
como alternativa para la superación de condiciones de vulnerabilidad y marginación.

De igual forma, se inició la primera etapa de implementación de una agenda interinstitucional ambiental con enfoque de género, como instrumento de gestión ambiental participativa que permite involucrar a los actores locales en la conservación y recuperación ambiental de su territorio y promover un manejo apropiado de los recursos naturales, así como de evidenciar, promover y potenciar el papel central de las mujeres en la gestión ambiental de su territorio.

En primer lugar, se describe la metodología empleada y se presentan algunos aportes que han surgido en el escenario académico en torno al empoderamiento de la mujer como condición del progreso y de la sostenibilidad rural. Luego, se señala en qué medida las AIA son instrumentos de gestión ambiental participativa y se ofrece una breve descripción del contexto local de la zona de estudio. Por último, se presentan los principales hallazgos y las reflexiones surgidas en torno al caso de estudio y los avances logrados con la implementación de la agenda interinstitucional ambiental con enfoque de género.

\section{Metodología}

Este artículo presenta los resultados de un proyecto de investigación desarrollado en el marco de un convenio de asociación entre la Universidad del Rosario de Colombia y la autoridad ambiental Corporación Autónoma Regional de Cundinamarca (CAR), ejecutado con el propósito de avanzar en el estudio de las agendas interinstitucionales ambientales como instrumentos de gestión basados en la promoción de la gobernanza ambiental local, la participación ciudadana, el empoderamiento comunitario y la coordinación entre los actores involucrados.

Para la implementación de esta propuesta, se eligió la vereda Llano Grande del municipio de Vergara debido a los particulares elementos que allí confluyen, como las persistentes condiciones de marginación y vulnerabilidad de su población, las consecuencias devastadoras del conflicto armado y la importante iniciativa de empoderamiento y organización de la población femenina en la reconstrucción de sus planes de vida. 
Se llevó a cabo una etapa de sensibilización, divulgación y diagnóstico de la situación local con respecto a las relaciones de género, la presión antrópica sobre los recursos naturales y las iniciativas que han surgido de las mujeres de la vereda para adoptar acciones comunitarias de sostenibilidad ambiental, para superar sus condiciones de marginación y para mejorar el uso, manejo y aprovechamiento de los recursos naturales. Esto se hizo con base en el modelo de Investigación-Acción Participativa (IAP), enfocado en promover la intervención y participación activa de los actores sociales en los procesos de investigación y en estimular la implementación de prácticas transformadoras del conflicto por medio del diálogo entre la academia y la comunidad (Rahman y Fals Borda, 1989).

Se partió de la recopilación de información acerca de las condiciones generales de la población y del territorio mediante la consulta de fuentes secundarias, la interacción directa con la población de la vereda, entrevistas semiestructuradas, entrevistas informales, observación participante y grupos focales; todo lo anterior con el fin de consolidar una línea base que permitiera comprender las particularidades del caso de estudio y fortalecer los lazos de confianza, comunicación y participación entre los actores comunitarios e institucionales de la gestión ambiental del territorio.

En segundo lugar, se hicieron entrevistas a profundidad y semiestructuradas a veinte miembros de la comunidad de la vereda Llano Grande, para obtener un primer diagnóstico acerca de los factores de deterioro ambiental que requieren atención prioritaria en la zona y del rol que las mujeres de la vereda han desempeñado en la gestión ambiental participativa de los recursos naturales de este territorio, así como en la superación de las condiciones de vulnerabilidad y marginación causadas por las desigualdades de género y el impacto del conflicto armado.

Asimismo, se hicieron tres reuniones de grupos focales con habitantes de la vereda y con mujeres pertenecientes a la asociación productiva Asoproliv, en las que hubo un espacio de intercambio de percepciones, oportunidades, motivaciones y experiencias acerca del empoderamiento de las mujeres rurales, la gobernanza ambiental con enfoque de género y su aporte a la adaptación y superación en un escenario de posconflicto. Del mismo modo, se llevaron a cabo recorridos vivenciales con estos grupos focales por algunas fincas y zonas de importancia hídrica, con el objetivo de retroalimentar, fortalecer y promover el interés por la gestión ambiental 
participativa de estas mujeres líderes y avanzar hacia la consolidación de un plan de trabajo conjunto que involucre a la comunidad de manera activa.

\section{La mujer, un actor protagónico en la transformación del sector rural}

En Colombia, muchas mujeres rurales han visto vulnerados sus derechos, al sufrir las consecuencias del conflicto armado y las relaciones de dominación y maltrato en razón al género (Guerra y Plata, 2005). El conflicto armado ha impactado de modo diferencial a mujeres, menores de edad y miembros de grupos étnicos, debido a sus especiales condiciones de vulnerabilidad y necesidad, pues estos grupos poblacionales deben superar mayores dificultades para sobrevivir y recuperar sus proyectos de vida destruidos por la violencia (Meertens, 2004).

Las mujeres son más vulnerables frente a la guerra, ya que, en gran medida, son víctimas de abuso sexual, secuestro, tortura, traumas morales y psicológicos. Esto, sumado a los roles de género que les han sido asignados culturalmente, como la responsabilidad del cuidado de los hijos o una posición de subordinación frente a los hombres, hace que sufran con mayor rigor las consecuencias de un conflicto armado. En muchas ocasiones, por ejemplo, como madres solteras se ven obligadas a asumir actividades productivas adicionales para suplir la ausencia de miembros del núcleo familiar y enfrentarse a mayores responsabilidades y nuevas condiciones de vida (Ahmed, 2007; Ruiz y Aldás, 2007).

En el sector rural persisten distinciones referentes a las responsabilidades, las funciones y los papeles asignados a hombres y mujeres en el uso y manejo de los recursos naturales, puesto que las ocupaciones y labores asociadas con el cuidado del hogar y de la familia suelen recaer sobre las mujeres, sin recibir alguna remuneración; mientras, la participación de los hombres en estas tareas es tangencial, porque según lo prescribe la tradición, les corresponde el trabajo que es retribuido económicamente fuera del hogar (Benítez et al., 2012). Además, existen marcadas desigualdades en la obtención de los recursos, insumos y servicios, lo que hace que en muchos casos las mujeres tengan que enfrentar mayores dificultades en el 
acceso a la tierra, a la educación y a servicios de crédito y subsidios (Croppenstedt, Goldstein y Rosas, 2013).

Lo anterior ha ubicado a la mujer rural en posición de vulnerabilidad y escasez de oportunidades de desarrollo y formación (Álvarez et al., 2012) y ha restringido su participación en la esfera pública a lo largo de la historia. Por ello, se requiere hacer esfuerzos para transformar las construcciones sociales en torno a las concepciones de género excluyentes e inequitativas (Mingol, 2007) y así avanzar hacia la equidad de género, en el sentido de buscar una mayor participación de las mujeres en las decisiones que las involucran y cada vez más inclusión de sus opiniones y propuestas (Portolés, 2007).

Ahora bien, aun cuando en el sector rural han sido muy marcadas las problemáticas socioeconómicas y las desigualdades de género, en las últimas décadas se han generado importantes cambios tanto en el desarrollo del sector rural como en la desarticulación de estructuras patriarcales (SánchezMuros y Jiménez, 2013). Aunque a las mujeres se les ha conferido un rol tradicional de víctimas en el contexto del conflicto armado y la pobreza del sector rural, ellas se han venido consolidando como nuevos sujetos sociales en el marco de las transformaciones del sector rural (Meertens, 2004), posicionadas como actores sociales visibles y autónomos en la escena productiva, comunitaria y familiar (Shmite, 2009).

A pesar de la marcada división del trabajo en el sector rural en razón del género, se ha observado que es común que los cónyuges posean un conocimiento y una perspectiva similar frente a los recursos naturales de su entorno y sus actividades de subsistencia, debido a que mediante las interacciones cotidianas se comparten información y puntos de vista. Lo anterior sugiere que el grado de influencia de las mujeres rurales en la adopción de decisiones enfocadas a la gestión sostenible de los recursos puede subvalorarse en muchos casos; además, se ha observado en diferentes latitudes que las mujeres asociadas lideran procesos de gestión ambiental muy valiosos (Elias, 2015).

También se ha evidenciado la potencialidad de las mujeres para la transformación negociada de los conflictos, derivada en parte del aprendizaje de la experiencia maternal, fuente de fortalecimiento del valor del cuidado. Esto corrobora la necesidad de considerar experiencias y saberes en una perspectiva de equidad de género en la transformación de conflictos 
(Albert, 2007; Mingol, 2007). Asimismo, se ha observado que la participación de las mujeres rurales en el trabajo con la comunidad puede generar y robustecer conocimientos, virtudes, sentido de pertenencia e identidad y sentimientos de solidaridad comunitaria en los demás miembros de la colectividad (Benítez et al., 2012).

De allí el concepto de empoderamiento, que se suele definir como el proceso mediante el cual se adquiere la capacidad de adoptar y participar en la toma de decisiones, cuando esta posibilidad había sido negada. El empoderamiento femenino está enfocado en lograr en las mujeres un mayor control y acceso sobre los recursos, una verdadera participación en las decisiones estratégicas que les involucran y mejores condiciones para la superación de las desigualdades de género (Casique, 2010).

El empoderamiento femenino, orientado a la promoción, al fortalecimiento y a la propagación de capacidades y potencialidades de las mujeres para participar, decidir, influir y actuar en beneficio de sus propias necesidades y las de su comunidad, genera notables resultados en materia de emprendimiento, desarrollo local y regional, dinamización social, erradicación de pobreza, aumento en la producción, generación de empleo y transformación de estructuras patriarcales (Buendía-Martínez y Carrasco, 2013).

En el marco de las iniciativas para reducir la pobreza en el sector rural, se ha evidenciado que la implementación de proyectos de subsistencia en los que se involucra la participación de los actores sociales locales trae consecuencias positivas en el empoderamiento de las mujeres dentro y fuera del hogar y en su participación en la adopción de decisiones (Khanna, Kochhar y Palaniswamy, 2015).

Por lo tanto, en un marco de conflicto y dificultades, las alternativas de supervivencia y reedificación comunitaria cobran un gran valor como estrategias de empoderamiento de las mujeres, por ejemplo, con la conformación de asociaciones y el fortalecimiento de la participación femenina en la toma de decisiones (Ahmed, 2007). La conformación de grupos o asociaciones comunitarias ha sido considerada como una manifestación avanzada de participación, en el sentido de que permite trascender las fases más básicas de participación -como la consulta o la información- hacia escenarios de comunicación, concertación y acción participativa. Esto surge de la apertura de canales de participación e influencia pública para aquellas mujeres que se habían visto relegadas al ámbito familiar y doméstico, con 
pocas oportunidades de educación, empleo y socialización, por un lado, y la generación de integración social y la promoción de la gestión de los intereses comunes y procesos de cambio, por otro (Prager, 2010; SánchezMuros y Jiménez, 2013).

Hay investigaciones que muestran que el surgimiento de empresas rurales domésticas compatibles con las actividades agrícolas tradicionales proporcionan mayor estabilidad económica y mejores condiciones de vida, por lo que se han convertido en la estrategia de muchas mujeres rurales para encontrar alternativas de mejoramiento de sus ingresos y superación de las condiciones históricas de discriminación y desigualdad de género (Álvarez et al., 2012). También se ha observado que existe un vínculo entre los recursos y el poder de decisión en la familia, pues las mujeres con posibilidades de acceder y controlar recursos económicos y sociales tienen una mayor capacidad de decisión dentro de sus hogares, así como aquellas mujeres propietarias de su vivienda y que cuentan con alguien que les ayuda a cuidar sus hijos tienen mayor probabilidad de afianzar su participación en la esfera pública (Casique, 2010).

Luego, para adquirir mayor independencia, las mujeres rurales requieren, entre otras cosas, apoyo del Gobierno, del sector privado y de las ONG en temas como el fortalecimiento de sus cooperativas y la asignación de presupuesto o insumos para su promoción (Arfaee, Rezaei y Zand, 2015).

Es importante robustecer la perspectiva de género en el abordaje de los conflictos ambientales del sector rural, con el objeto de resaltar y reivindicar los roles de las mujeres en el tratamiento y la resolución o transformación de dichos conflictos, puesto que este grupo poblacional ha sido vulnerado históricamente y ahora es pertinente reforzar su concepción como sujeto de derechos y como actor fundamental en la conservación del ambiente y de los recursos naturales, a partir de la promoción de su participación en las decisiones que le afectan y la garantía de la igualdad de género en el momento de planear y adoptar medidas frente a los conflictos ambientales.

\section{Ruralidad y ambiente}

El impacto negativo sobre el ambiente por parte de las actividades humanas ocasiona una constante vulneración y amenaza a los derechos 
humanos y propicia el surgimiento de conflictos socioambientales, que adquieren mayor complejidad en el marco de concepciones antropocéntricas de desarrollo que no consideran como prioritaria la protección y recuperación de los recursos naturales. Es necesario, pues, avanzar en la creación, el perfeccionamiento y la aplicación de nuevas formas de progreso basadas en la sustentabilidad y la equidad y validar el papel fundamental que desempeñan las mujeres en este proceso (Rico, 1998).

Ante las condiciones de degradación ambiental e inadecuado uso y manejo de los recursos naturales en el sector rural, es preciso ir hacia la consolidación de sistemas de producción que incorporen el respeto y la preservación del ambiente como valores fundamentales, de modo que se pueda garantizar la disponibilidad presente y futura de los recursos naturales y fortalecer la organización de los actores locales para aumentar su competitividad en los mercados (Villa y Bracamonte, 2013).

Los esquemas basados en el respeto por el conocimiento local, el empoderamiento de las comunidades y el manejo sostenible de los recursos naturales han tenido gran acogida en países como Australia, en donde se han promovido los grupos de cuidado de la tierra locales como mecanismos para incrementar la participación de las comunidades en la gestión ambiental de su territorio, con objetivos comunes (Prager, 2010). La Comisión Europea ha formulado estrategias para un crecimiento inteligente, sostenible e integrador, construidas a partir de elementos como el conocimiento, la innovación, la eficiencia en el uso de recursos, el desarrollo de prácticas sostenibles y el fomento de la cohesión social (European-Commission, 2010).

Se han identificado algunos campos de acción indispensables para afrontar escenarios de posconflicto, entre los que se encuentran la promoción y el fortalecimiento de alternativas de progreso sostenible para los sectores rural y urbano, la consolidación del diálogo como mecanismo de transformación de conflictos y adopción de decisiones y la incorporación de valores como la solidaridad, la comunicación y la colaboración como parte de la cultura (Guerra y Plata, 2005). Por lo tanto, el surgimiento de actividades emprendedoras en el sector rural es un elemento fundamental para alcanzar metas relacionadas con la erradicación de la pobreza y el manejo sostenible de los recursos naturales, proceso en el que las mujeres tienen un llamado a participar activamente, por ejemplo, mediante su aporte 
a la diversificación y el enriquecimiento de las actividades productivas de subsistencia (Buendía-Martínez y Carrasco, 2013).

Por su parte, las agendas interinstitucionales ambientales (AIA) pueden definirse como instrumentos de diagnóstico, participación y gestión ambiental en los que se abren canales de comunicación, concertación e intercambio de conocimientos y experiencias entre los actores, para dirimir o disminuir la intensidad de los conflictos de carácter socioambiental a partir de la gobernanza ambiental. Estas agendas constituyen un plan integral de trabajo, elaborado de manera conjunta mediante la interacción entre las instituciones del Estado, la sociedad civil organizada y el sector privado, en función de la protección del ambiente, del empoderamiento comunitario y del desarrollo humano sostenible (Güiza y Palacios, 2014).

Lo anterior responde a la necesidad de superar la tradicional y unidireccional manera de gobernar, en la que el Estado es el actor central y los actores sociales simples sujetos pasivos o destinatarios de las políticas públicas (Aguilar, 2010). De ahí han surgido conceptos como el de gobernanza ambiental, que implica la participación directa de los actores comunitarios en la concertación y adopción de decisiones relacionadas con la gestión ambiental de su territorio, de modo que a partir de su participación voluntaria y aportes en materia de recursos, iniciativas y conocimiento, se obtenga una influencia positiva en el resultado y la efectividad de las políticas públicas, ya sea como consecuencia de su incorporación dentro del sistema de hábitos y prácticas comunitarias o por su formalización con reglamentos comunitarios (Gasca Zamora, 2014; Haldrup, 2015).

En el campo de la organización comunitaria, han surgido conceptos como el de subculturas agrícolas, como un conjunto de prácticas agroambientales arraigadas en las comunidades rurales, que responden a un sistema de normas y valores comúnmente aceptados e incorporados en el imaginario colectivo. El surgimiento y fortalecimiento de estas subculturas propicia una especie de control social frente a las conductas que no son compatibles con los valores de conservación ambiental grupalmente aceptados (Taylor y Van Grieken, 2015) y una alta aceptación y buen reconocimiento ante los miembros de la comunidad que implementen buenas prácticas agrícolas (Burton, Kuczera y Schwarz, 2008).

Cabe resaltar que la renuencia frente a la implementación de acciones de conservación en las poblaciones rurales, en algunos casos, se puede 
explicar por la falta de una ventaja visible inmediata en términos económicos. Esto puede contrarrestarse con la adopción de enfoques de gestión ambiental construidos en torno a la participación de las comunidades locales, lo que, a su vez, provoca una fuerte motivación de colaboración voluntaria entre los actores sociales involucrados (Marshall, 2009).

Todo lo anterior permite entender por qué, en materia de gestión ambiental rural, el concepto de gobernanza es cada vez más relevante. Este último permite adoptar perspectivas integrales que abarquen los factores ecológicos, económicos, sociales y culturales involucrados, de manera que las políticas ambientales bajo este enfoque adquieren una connotación especial, al instaurar, promover y fortalecer la sostenibilidad ambiental en las actividades productivas y las prácticas sociales y culturales de la comunidad (Funtowicz y Ravetz, 1993; Rodríguez-Becerra, Espinoza y Wilk, 2002) y posibilitar la implementación de mecanismos transparentes, participativos e incluyentes de planeación, seguimiento y control ambiental, que faciliten una acción coordinada y complementaria entre las entidades estatales y los actores sociales (Paré y Fuentes, 2007).

La construcción e implementación de una AIA es una alternativa para avanzar hacia modelos de gestión pública basados en la gobernanza ambiental, pues se abren escenarios de intercambio de conocimientos y experiencias y se propician la participación comunitaria y la coordinación de múltiples actores, lo que permite la materialización de derechos humanos mediante la prevención, mitigación y transformación de los conflictos ambientales (Güiza y Palacios, 2014). Lo anterior surge porque la implementación de programas agroambientales que involucran a los actores, incluso colectivos y asociaciones comunitarias, genera relaciones de confianza, comunicación y cooperación que permiten la evolución de proyectos voluntarios de prácticas de conservación en el sector rural (Taylor y Van Grieken, 2015).

Los lazos de confianza y las buenas relaciones entre los actores involucrados en la gestión agroambiental rural influyen directamente en el grado de compromiso local para invertir tiempo y trabajo en la implementación y el desarrollo de este tipo de proyectos, pues media una expectativa de resultados benéficos (Mettepenningen, Beckmann y Eggers, 2011). Además, la aceptación de nuevos modelos de producción rural sostenible depende en gran medida de la confianza que los actores comunitarios locales sientan 
hacia los actores institucionales y privados externos que pretenden participar y promover estos esquemas, porque se requiere confianza y credibilidad en el conocimiento y las propuestas que se comparten (Carolan, 2006).

Por otro lado, se ha observado que abrir y fortalecer canales de diálogo entre los actores brinda facilidades para la coordinación y disminuye el desequilibrio de poder ocasionado por la posesión unilateral de la información (Prager, 2010). Esto último cobra gran importancia en las zonas rurales apartadas geográficamente, donde suelen presentarse obstáculos frente al acceso de conocimiento externo y hay pocas alternativas significativas para el intercambio de conocimientos (Naldi, Nilsson, Westlund y Wixe, 2015).

\section{Pobreza, conflicto armado y mujer rural en la zona de estudio}

El municipio de Vergara, ubicado en el departamento de Cundinamarca, sobre la cordillera Oriental de Los Andes, posee un $82 \%$ de territorio montañoso y quebrado. Su temperatura oscila entre los 15 y los 25 grados centígrados y la precipitación, en un rango de 1.800 a $2.200 \mathrm{~mm}$ anuales. La actividad económica se centra en el cultivo de café y caña panelera y, en menor medida, de maíz, plátano, yuca, hortalizas, frutales y pastos para ganadería (Alcaldía Municipal de Vergara, 2010).

El territorio de Vergara se encuentra dividido en 29 veredas y cuenta con una población estimada de 7.664 habitantes, de los cuales un 52,1\% es hombre y un $47,8 \%$, mujer; un $28,4 \%$ proviene de otro municipio y un $17,9 \%$ presenta alguna limitación permanente. El analfabetismo es de $12,3 \%$ entre la población de 5 años y más y de un 13,7\% en personas de 15 años y más. La vereda Llano Grande es una de las que cuenta con mayor población, ya que tiene alrededor de 134 familias compuestas por cerca de 512 miembros en total. Un 76,2\% de los hogares de Vergara está compuesto por un núcleo familiar de cuatro o menos personas. Un 81,8\% de las viviendas del municipio cuenta con conexión a energía eléctrica y en $89,1 \%$ de las viviendas rurales se desarrollan actividades agropecuarias discriminadas de la siguiente manera: actividad agrícola, 76,4\%; pecuaria, $92,0 \%$ y piscícola, $9,0 \%$, con la particularidad de que en la mayoría de los hogares se ejecutan dos o tres tipos de actividades. Respecto a la 
actividad agrícola, se estableció que del total de cultivos registrados en las zonas rurales, un 31,8\% corresponde a cultivos transitorios solos; un $7,2 \%$ a transitorios asociados; un $46,2 \%$ a cultivos permanentes solos y un $14,8 \%$ a permanentes asociados (Departamento Administrativo Nacional de Estadística, 2005).

Ahora bien, el conflicto armado ha ocasionado condiciones de desigualdad e injusticia social en la población, con mayor intensidad en las zonas rurales, en donde la violencia, las debilidades en el sistema de salud, las deficiencias en el suministro de los servicios públicos y las condiciones de pobreza son el común denominador (Angulo y Luque, 2008). Se calculó que para el año 2005, cerca de un $70 \%$ de las víctimas sobrevivientes del conflicto armado era mujer; más de 110.000 mujeres que, además de la desigualdad, discriminación y agresión que han sufrido en razón de su género, han tenido que soportar el inclemente peso de la guerra y sus consecuencias (Pérez, 2005). Según manifestaron algunos líderes encuestados, en Vergara el conflicto armado tuvo una gran intensidad entre los años 1997 y 2005, período en el que se presentaron acciones violentas que involucraron a diferentes actores, en especial grupos paramilitares, situación que causó asesinatos selectivos y enfrentamientos armados que dejaron a su paso una gran victimización (M. A. Díaz, comunicación personal, 4 de junio de 2015).

Puesto que el conflicto armado en Colombia ha afectado en mayor medida al sector rural, es necesario involucrar de manera activa a las comunidades rurales en el diseño de medidas para superar las consecuencias de violencia, en especial a las mujeres, quienes sufren de modo diferencial la violencia y la marginación (Romero, 2012). En el país persisten condiciones de desigualdad, discriminación, exclusión y violencia hacia las mujeres, sobre todo en el ámbito educativo, laboral y político y, en gran medida, son el blanco de la violencia sexual, intrafamiliar y del desplazamiento (Angulo y Luque, 2008).

Aunque según cifras del Programa de las Naciones Unidas para el Desarrollo (PNUD, 2012) los índices de pobreza han disminuido en el departamento de Cundinamarca, el porcentaje de familias que sobrevive con ingresos bajo la línea de pobreza es preocupante: cerca de 25,3\% para el año 2010 y casi un 13,2\% de la población presenta necesidades básicas insatisfechas. 
Parte del territorio del departamento de Cundinamarca ha sido escenario de disputa armada por parte de grupos militares, paramilitares y guerrilleros, lo que ha generado presión negativa sobre la población rural y la vulneración progresiva de sus derechos humanos (Pérez, 2005).

Los grupos guerrilleros, en especial las Fuerzas Armadas Revolucionarias de Colombia (FARC), han estado presentes en el departamento de Cundinamarca desde su surgimiento en la década del cincuenta, sobre todo en los municipios cercanos a Bogotá. Desde 1982, en la Séptima Conferencia de las FARC, Cundinamarca fue señalado como punto estratégico de sus acciones armadas, por lo que se planteó como objetivo que el grupo insurgente consolidaría su presencia en dicho departamento para el año 2000 por medio de numerosas estructuras como los Frentes 22, 42, 25 y 52, entre otros (Misión de Observación Electoral [MOE], s. f.). El Frente 22 incidió de manera directa en el municipio de Vergara.

Por otro lado, los grupos paramilitares o de autodefensa han estado en el departamento de Cundinamarca desde la década del ochenta en municipios como San Cayetano, Vergara, Yacopí, Paime, El Peñón, Pacho, La Palma, Puerto Salgar y Guaduas. Su accionar estuvo relacionado con las actividades de los narcotraficantes y su interés por los corredores geográficos. Los grupos de autodefensas se consolidaron bajo el nombre de Autodefensas Campesinas de Cundinamarca.

Estos dos actores armados ilegales tuvieron enfrentamientos intensos desde el año 2003 en todo el departamento hasta el año 2004, cuando el bloque Cundinamarca se desmovilizó en el marco de las negociaciones entre el Gobierno nacional y las Autodefensas Unidas de Colombia (AUC) (MOE, s. f.).

Lo anterior ha tenido nefastas consecuencias en la población de las zonas que han sido objeto de disputa por el dominio territorial (Vicepresidencia de la República de Colombia, 2001). La presencia y el impacto de los grupos paramilitares en el departamento de Cundinamarca, incluidos Vergara y Bogotá, se vivieron con mayor intensidad a mediados de la década del noventa, lo que coincidió con la reubicación de muchos cultivos ilícitos del sur del país y el fortalecimiento de la presencia de las FARC en el departamento (Pérez, 2005).

Los grupos paramilitares se han caracterizado por el empleo de métodos muy crueles de hostigamiento a la población, como asesinatos colectivos 
y torturas; tanto así, que entre los años 1990 y 2000, en Cundinamarca se registraron 370 vidas humanas perdidas en eventos de asesinatos colectivos con cuatro o más víctimas. Además, fueron responsables de $90 \%$ del total de 1.563 homicidios violentos perpetrados por grupos armados en Cundinamarca en la década del noventa (Vicepresidencia de la República de Colombia, 2001).

Aunque el conflicto armado en Colombia ha perdurado por más de cinco décadas, es muy escasa la literatura sobre las estrategias de supervivencia de las víctimas de la violencia en el sector rural (Fernández, Ibáñez y Peña, 2014).

\section{Resultados y discusión}

La participación ciudadana con enfoque de género y el empoderamiento femenino son conceptos que cobran cada vez más vigencia en la vereda Llano Grande, pues han empezado a surgir asociaciones de mujeres rurales víctimas de la violencia, la desigualdad socioeconómica y la exclusión de género, que buscan fortalecerse con un enfoque productivo y ambiental.

Tal es el caso de la Asociación de Productoras Líderes de Vergara (Asoproliv), conformada por mujeres productoras de café y, en su mayoría, víctimas del conflicto armado, en particular por el accionar de grupos paramilitares. Muchas mujeres de este territorio perdieron integrantes de su familia y se vieron obligadas a hacerse cargo de sus familias.

Lo anterior, sumado a las condiciones de marginación, pobreza y desigualdad, llevó a que mujeres líderes de la población se unieran con el objetivo común de conformar una asociación productiva que les permitiera superar las condiciones de vulnerabilidad y mejorar el desarrollo local de su comunidad.

Así lo expresaron algunas mujeres líderes que participaron en el desarrollo de los grupos focales: "Cuando se acabó la guerra, muchas de nosotras quedamos viudas, con hijos, al frente del hogar; entonces decidimos conformar la asociación para buscar mejores condiciones de vida y salir adelante" (A. L. Zárate, comunicación personal, 4 de junio de 2015).

Las mujeres líderes de Asoproliv reconocen que aunque la asociación lleva dos años desde su formalización legal y ha recibido poco apoyo de 
actores externos, a partir de su conformación como grupo, hace más de diez años, hasta la actualidad, han observado resultados positivos por el solo hecho de haberse unido para conformar el grupo. Resaltan la promoción de valores como la solidaridad y la cooperación entre los miembros de la comunidad, el fortalecimiento de los vínculos de comunicación y confianza entre los mismos, el bienestar y la motivación emocional y la generación de identidad en torno al grupo y su labor.

La participación ciudadana manifestada en la asociación de estas mujeres ha permitido hacer frente a las históricas desigualdades ocasionadas por la fuerte influencia de las estructuras patriarcales en las relaciones de género, evidenciadas desde la limitación de sus labores al ámbito del hogar, la nula o escasa participación en las decisiones que las involucran tanto en la esfera familiar como pública, la sobrecarga de responsabilidades y la ausencia de oportunidades de formación y progreso y el maltrato, lo que es común en Colombia y otras latitudes (Ahmed, 2007; Benítez et al., 2012; Croppenstedt et al., 2013; Meertens et al., 2004; Ruiz y Aldás, 2007; Álvarez et al., 2012). Teresa Ríos, miembro de la asociación, señaló en el desarrollo de uno de los grupos focales:

Hace unos años había en nuestra comunidad mucho machismo y discriminación. Las mujeres no participábamos en nada y solo podíamos dedicarnos al cuidado del hogar y los niños, además de que nuestra mano de obra siempre la han pagado más barata, aunque tenemos más cargas y responsabilidades que los hombres (T. Ríos, comunicación personal, 15 de junio de 2015).

La conformación de la asociación de mujeres ha permitido que sus miembros lleven a cabo la siembra, cosecha, el tostado, molido y la comercialización de su propia marca de café como medio de subsistencia para sus familias. También ha fomentado profundas transformaciones en las relaciones de género presentes en la vereda y el municipio, pues les ha abierto a estas mujeres nuevos canales de participación y comunicación con los miembros de su comunidad y los actores institucionales con jurisdicción en el territorio, ya que se han involucrado en la gestión ambiental de su entorno y han canalizado el apoyo para mejorar la sostenibilidad de las actividades productivas de la vereda. 
En los últimos dos años, las mujeres miembros de Asoproliv han adelantado y participado en procesos de capacitación e implementación de métodos orgánicos sostenibles en la cultivo del café, de reciclaje para la producción de fertilizantes con residuos orgánicos, actividades de intercambio de saberes y experiencias de sostenibilidad productiva y están dando los primeros pasos en la conformación de huertas caseras orgánicas; esto constituye un importante avance para la reducción de la contaminación y los impactos ambientales y en la salud por el uso de agroquímicos.

Estas mujeres líderes han demostrado una notable conciencia ambiental de conservación de los recursos naturales, por lo que se han convertido en generadoras y replicadoras de concepciones de sostenibilidad y liderazgo ambiental desde sus núcleos familiares hasta sus relaciones con los demás miembros de la comunidad. Como habitantes del territorio, han observado que la riqueza hídrica de la vereda se ha visto afectada a consecuencia del impacto de la actividad agrícola y ganadera sobre zonas de especial importancia ambiental en donde nacen numerosos arroyos y quebradas y que, a pesar de su vocación protectora, han sido deforestadas y reemplazadas por potreros para la ganadería y cultivos de café y caña de azúcar, lo cual pone en un serio riesgo la permanencia de estas fuentes de agua.

Así lo reiteraron algunas líderes de la Asociación: "Lo que queremos a futuro es trabajar, pero conservar; si queremos tener buenas condiciones de vida, necesitamos hacer que la ganadería y la agricultura no sean dañinas, más que todo para el agua" (A. L. Zárate, comunicación personal, 4 de junio de 2015).

Por otro lado, un $80 \%$ de los encuestados reconoció que las medidas sobre estos nacimientos de agua son necesarias y urgentes, porque en los últimos veinte años se ha apreciado una significativa diminución en la oferta hídrica de estos arroyos, en especial durante las épocas de sequía, que cada vez son más intensas y largas. Por ello, algunas familias suelen tener dificultades por la escasez del recurso, lo que no ocurría antes.

Según resaltaron algunas mujeres líderes, tradicionalmente, las decisiones relacionadas con el uso y aprovechamiento de los recursos naturales para el desarrollo de actividades productivas correspondían al género masculino, aun cuando en la cotidianidad las mujeres perciben y afrontan las consecuencias negativas de un actuar poco planificado, en particular sobre el recurso hídrico, fundamental para la satisfacción de las necesidades 
en cada hogar. Sin embargo, hoy en día es posible ver que las mujeres del municipio son protagonistas en materia de gestión ambiental local y se han apropiado de sus capacidades como actores clave en la conservación del agua.

De la mano con miembros de la Junta de Acción Comunal, habitantes de la vereda y la CAR, Asoproliv ha liderado procesos de recuperación y aislamiento de las rondas de protección hídrica de nacimientos, arroyos y quebradas en tres veredas de su municipio, así como de arborización mediante cerca viva protectora-productora y frutales en fincas de la vereda, implementación de mejoras en la diversidad alimenticia e investigación vegetal de especies alimenticias protectoras y prácticas regeneradoras de suelos.

La intención y voluntad de adelantar trabajos de cercado y reforestación de zonas estratégicas para la conservación del recurso hídrico es generalizada por parte de la población beneficiaria del agua y el papel de las mujeres de la asociación es fundamental. Sin embargo, no se ha obtenido colaboración de algunos propietarios de los predios en donde se ubican los nacimientos y las corrientes de agua. Además, las integrantes de Asoproliv han manifestado la necesidad de apoyo externo en materia de recursos y formación para continuar con el fortalecimiento de su organización y la superación de sus condiciones de vulnerabilidad.

Se evidenció la existencia de un desacuerdo con respecto al uso de suelo de las zonas periféricas a las fuentes de agua, porque algunos propietarios han manifestado su inconformidad acerca de la instalación de cercas que delimiten la ronda hídrica de protección y la excluyan de la actividad ganadera y agrícola, con el fin de reforestar estas áreas con vegetación nativa. Por esta razón, el impacto continúa causándose y no se ha logrado un acuerdo para tomar medidas de mitigación, restauración y protección.

Un 40\% de los encuestados manifestó que la falta de consenso con los propietarios de los predios que colindan con los nacimientos y arroyos se debe a la desconfianza en cuanto a la propiedad de la tierra que se destina a la conservación. Por lo tanto, en la primera etapa de diseño e implementación de la agenda interinstitucional con enfoque de género, además de un diagnóstico participativo, se llevó a cabo un proceso de sensibilización y divulgación en torno al conflicto ambiental relacionado con el impacto sobre el recurso hídrico. Con la colaboración activa de las mujeres líderes de Asoproliv (en los recorridos, las entrevistas y los grupos focales), se abrieron 
escenarios de intercambio, socialización y reflexión acerca de la importancia de hacer esfuerzos por mejorar las prácticas y los hábitos comunitarios de conservación ambiental.

Luego de generar y fortalecer los canales de confianza y diálogo entre los miembros de la comunidad, con la protagónica labor de las mujeres líderes de Asoproliv en conjunto con los miembros de la comunidad directamente implicados en la afectación del agua, se constituyó una serie de compromisos como la participación activa en la conservación del recurso hídrico; la recuperación y protección de las áreas de ronda de nacimientos, arroyos, quebradas, humedales y demás cuerpos de agua; la no tala o afectación de la vegetación nativa que se mantiene en las rondas de cuerpos de agua y formar parte de las jornadas comunitarias de reforestación, aislamiento y mantenimiento de las zonas de protección hídrica que se definan como prioritarias.

Durante la implementación de la agenda interinstitucional, las mujeres de la Asociación demostraron sus capacidades de liderazgo y fueron protagonistas en los escenarios participativos y de intercambio, convertidas en actores clave en materia de comunicación, negociación y construcción de acuerdos con los actores involucrados.

Los propietarios de predios estratégicos para la conservación de nacimientos, arroyos, quebradas, humedales, lagos y demás cuerpos de agua que se encuentran en riesgo se comprometieron a cumplir su deber de permitir y contribuir a hacer las tareas de reforestación y cercado para la protección del recurso hídrico, así como a conservar las áreas reforestadas y los trabajos adelantados en beneficio de la comunidad.

La comunidad manifestó la necesidad de pedir apoyo a los actores institucionales en lo que se refiere al suministro de materiales para el cercado de las áreas periféricas a los cuerpos de agua, priorizados, de árboles nativos que conserven el agua para la reforestación en las zonas de protección del recurso hídrico y de materiales para la construcción de bebederos para el ganado que pastorea en dichos predios.

Se constituyó el Comité de Gestión y Seguimiento conformado por miembros de la vereda, al cual se le otorgó la función de verificar el cumplimiento de los compromisos adquiridos y de promover la planeación, implementación y evaluación de planes de acción que garanticen el desarrollo sostenible de la vereda. 
Con base en las propuestas, las percepciones y los aportes de la comunidad involucrada se identificaron tres líneas de acción fundamentales para avanzar en el fortalecimiento de la gobernanza ambiental local: la recuperación y conservación de zonas de protección hídrica, la sostenibilidad productiva, la participación ciudadana y la educación ambiental.

Para desarrollar estas líneas de acción, se identificó una serie de acciones prioritarias por parte de los miembros de la comunidad de la vereda, como las labores de reforestación y aislamiento de zonas estratégicas para la conservación del recurso hídrico, la promoción del establecimiento de reservas naturales de la sociedad civil, actividades de formación y capacitación comunitaria para fortalecer la sostenibilidad productiva y reducir los impactos en los recursos naturales por las actividades agropecuarias, la recuperación de semillas y especies vegetales nativas, la implementación de semilleros familiares y escolares, capacitaciones, charlas, talleres y actividades participativas rurales relacionadas con la importancia de la conservación ambiental, el ahorro y cuidado del agua y el empoderamiento comunitario con enfoque de género.

Así pues, los mecanismos de participación ambiental que promuevan la comunicación entre los actores de diferentes sectores, además de generar lazos de confianza y cooperación y disminuir la brecha causada por la posesión unilateral de la información y las dificultades de las zonas rurales apartadas para el intercambio de información y nuevos conocimientos (Naldi et al., 2015; Prager, 2010; Taylor y Van Grieken, 2015), posibilitan rescatar el conocimiento tradicional y promover el reconocimiento y fortalecimiento de capacidades y potencialidades comunitarias y, de ese modo, avanzar hacia la construcción de modelos de gobernanza ambiental.

En el mismo sentido, el surgimiento de espacios que permitan el intercambio de percepciones, conocimientos, propuestas y experiencias facilita la identificación y construcción conjunta de alternativas productivas y de sostenimiento para contrarrestar las desigualdades en la demanda y remuneración de la mano de obra femenina.

Por esta razón, es fundamental incentivar la conformación de asociaciones que reivindican el rol de las mujeres campesinas en la conservación del ambiente y la producción sostenible, pues se evidencia que a partir de la asociación y cooperación comunitaria pueden ejecutarse proyectos que garanticen una calidad de vida digna mediante el empoderamiento de las 
mujeres y la promoción de su papel en la conservación y recuperación ambiental.

La promoción de iniciativas comunitarias de gestión ambiental puede robustecer el interés y la comprensión de la necesidad de adoptar prácticas sostenibles en los demás miembros de la comunidad, de modo que el crecimiento inteligente y el adecuado uso y la conservación de los recursos naturales se consoliden como elementos fundamentales en el plan de vida comunitario.

En consecuencia, las iniciativas comunitarias de conservación, aunadas a la organización mediante asociaciones productivas y Juntas de Acción Comunal, abren un panorama de posibilidades y potencialidades de construcción de un programa que refuerce la gobernanza y gestión ambiental comunitaria en el plano local y se incorporen la conservación ambiental y la sostenibilidad en las actividades productivas como elementos integrales del plan de vida comunitario.

\section{Conclusiones}

En un escenario de posconflicto, la promoción de la conformación de asociaciones comunitarias en torno a la producción sostenible puede traer resultados positivos para la superación de las consecuencias de la violencia y las desigualdades del sector rural, a partir del empoderamiento con enfoque de género y la consolidación de modelos de gestión pública basados en el concepto de gobernanza.

Puesto que la protección del recurso hídrico es un deber que corresponde a todos los actores, es menester construir escenarios en donde la participación ciudadana desempeñe un rol protagónico, con el fin de reunir los actores, establecer canales de diálogo entre ellos y construir en conjunto una ruta de acción que involucre el compromiso de todas las partes.

Cabe resaltar que el papel de liderazgo y emprendimiento de las mujeres en esta vereda es sobresaliente, ya que es común que se adelanten iniciativas de gestión ambiental e implementación de sistemas productivos sostenibles con una amplia participación y cooperación femenina, lo que permite vislumbrar las inmensas posibilidades de construcción de un tejido social y económico sostenible que favorezca la equidad de género. 
Las mujeres pueden contribuir a que los miembros de la comunidad comprendan y reafirmen la idea de que la protección del recurso hídrico y los recursos naturales renovales, así como la conservación de las zonas de protección hídrica, en general, es un deber y, a la vez, un derecho de rango constitucional y legal de obligatorio cumplimiento, de manera que el sinónimo de los compromisos y la ejecución de las acciones prioritarias surja de forma voluntaria. El empoderamiento femenino rural puede tener impactos positivos en la conservación ambiental del territorio y ser una estrategia pública de gestión ambiental local.

La participación ambiental, manifiesta en la conformación de asociaciones comunitarias, constituye un elemento esencial para el fortalecimiento de la gestión ambiental local y la reivindicación y el empoderamiento de la mujer campesina.

\section{Referencias}

Aguilar, L. (2010). Gobernanza: el nuevo proceso de gobernar: Recuperado de https://www.google.com.co/url?sa=t\&rct=j\&q=Eesrc=s\&source=web\&c $\mathrm{d}=1 \mathrm{G} \mathrm{cad}=$ rja\&uact $=8 \mathrm{Eved}=0 \mathrm{ahUKEwjYnYOpr} 4 \mathrm{LMAhWBth} 4 \mathrm{KHY}$ ObCVgQFggaMAA\&url=http\%3A\%2F\%2Fwww.la.fnst.org\%2Findex. php\%2Fbiblioteca-de-la-libertad\%2Fbiblioteca-de-la-libertad-en-americalatina\%2Fitem\%2Fdownload\%2F24_c4e2ee47bcbb24830a97e395eb340d 2f\&usg=AFQjCNGluMN89hbCefwZAdXEWTEy79tsmg\&sig2=7wEZvWY TVNP15V9qQ_nswEbvm=bv.119028448,d.dmo

Ahmed, F. (2007). Women and Conflict Transformation in Africa. Feminismo/s, 9, 67-78. Recuperado de http://rua.ua.es/dspace/handle/10045/3641

Albert, S. (2007). Reconstruir la identidad social de las mujeres para la transformación pacífica de los conflictos. Feminismo/s, 9, pp. 107-120. Recuperado de http://rua.ua.es/dspace/handle/10045/3644

Alcaldía Municipal de Vergara. (2010). Formulación del Plan de Desarrollo municipio de Vergara, departamento de Cundinamarca 2010-2011. Recuperado de http:// vergara-cundinamarca.gov.co/apc-aa-files/346332363534376437303666 62346235/Plan_de_Desarrollo_Vergara_2010_2011.pdf

Almeyra, G. (2012). Los cuatro jinetes del mundo rural latinoamericano. ALASRU, 6, pp. 13-23. 
Álvarez, Y., Díaz, R. y Saiz, J. (2012). Empresas de familia rurales, relaciones de género, relaciones de poder. Caso Lenguazaque, Cundinamarca. Criterio Libre, 10(17), pp. 53-72.

Angulo, C. y Luque, J. (junio, 2008). Panorama internacional de los derechos humanos de la mujer: una mirada desde Colombia. Revista de Derecho, 29, pp. 23-54. Arfaee, M., Rezaei, S. y Zand, A. (2015). Analyzing Environmental of the Rural Women Cooperatives and Their Share in the Agricultural Production in Tehran Province through SWOT Method. Mediterranean Journal of Social Sciences, 6(3), 518-523. doi:10.5901/mjss.2015.v6n3s2p518

Benítez, B., Dominí, M., Pino, M., Hernández, L., Yong, A., Medina, L. y Dueñas, F. (2012). Investigación participativa con enfoque de género. Logros de las mujeres de la provincia Mayabeque en el desarrollo local de sus patios y fincas. Cultivos Tropicales, 33(1), pp. 57-64.

Buendía-Martínez, I. y Carrasco, I. (2013). Women, Entrepreneurship and Rural Development in Latin America and the Caribbean. Cuadernos de Desarrollo Rural, 10(72), pp. 21-45.

Burton, R., Kuczera, C. y Schwarz, G. (2008). Exploring Farmers' Cultural Resistance to Voluntary Agri-environmental Schemes. Sociologia Ruralis, 48(1), pp. 16-37. doi: http://onlinelibrary.wiley.com/enhanced/doi/10.1111/j.14679523.2008.00452.x

Carolan, M. (2006). Social Change and the Adoption and Adaptation of Knowledge Claims: Whose Truth do you Trust in Regard to Sustainable Agriculture? Agriculture and Human Values, 23(3), 325-339. Recuperado de https://www. researchgate.net/publication/227225982_Social_Change_and_the_Adoption_and_Adaptation_of_Knowledge_Claims_Whose_Truth_Do_You_Trust_ in_Regard_to_Sustainable_Agriculture

Casique, I. (2010). Factores de empoderamiento y protección de las mujeres contra la violencia. Revista Mexicana de Sociología, 72(1), pp. 37-71.

Croppenstedt, A., Goldstein, M. y Rosas, N. (2013). Gender and Agriculture: Inefficiencies, Segregation, and Low Productivity Traps. World Bank Research Observer, 28(1), pp. 79-109. Recuperado de https://www.researchgate.net/profile/Andre_Croppenstedt/publication/256048594_Gender_ and_Agriculture_Inefficiencies_Segregation_and_Low_Productivity_Traps/ links/5587b9ec08aef58c03a04fc1.pdf

Departamento Administrativo Nacional de Estadística. (2005). Boletín Censo General 2005. Perfil Vergara-Cundinamarca. Recuperado de http://www.dane.gov.co/ files/censo2005/perfiles/cundinamarca/vergara.pdf 
Departamento Nacional de Planeación. (2013). Equidad de género para las mujeres, Conpes social 161. Recuperado de https://colaboracion.dnp.gov.co/CDT/ Conpes/Social/161.pdf

Elias, M. (2015). Gender, Knowledge-sharing and Management of Shea (Vitellaria Paradoxa) Parklands in Central-west Burkina Faso. Journal of Rural Studies, 38, 27-38. Recuperado de http://www.bioversityinternational.org/e-library/publications/detail/gender-knowledge-sharing-and-management-of-shea-vitellariaparadoxa-parklands-in-central-west-burkina-faso/

European-Commission. (2010). Europe 2020: A Strategy for Smart, Sustainable and Inclusive Growth: Communication from the Commission. Bruselas: Autor.

Fernández, M., Ibáñez, A. y Peña, X. (2014). Adjusting the Labour Supply to Mitigate Violent Shocks: Evidence from Rural Colombia. Journal of Development Studies, 50(8), pp. 1135-1155. Recuperado de http://www.tandfonline.com/ toc/fjds20/50/8

Funtowicz, S. y Ravetz, J. (1993). Science for the Post-normal Age. Futures, 25(7), 739-755. Recuperado de http://www.uu.nl/wetfilos/wetfil10/sprekers/Funtowicz_Ravetz_Futures_1993.pdf

Gasca Zamora, J. (2014). Gobernanza y gestión comunitaria de recursos naturales en la Sierra Norte de Oaxaca. Región y sociedad, 26(60), pp. 89-120. Recuperado de http://www.scielo.org.mx/scielo.php?script=sci_arttext\&pid $=$ S1870-39252014000300004

Guerra, M. y Plata, J. (2005). Estado de la investigación sobre conflicto, posconflicto, reconcialiación y papel de la Sociedad Civil en Colombia. Revista de estudios sociales (21), pp. 81-92. Recuperado de http://res.uniandes.edu.co/ view.php/461/index.php?id=461

Güiza, L. y Palacios, M. (2014). Construcción de agendas interinstitucionales ambientales: una mirada desde los derechos humanos. Bogotá: Universidad del Rosario.

Haldrup, N. (2015). Agreement Based Land Consolidation - In Perspective of New Modes of Governance. Land Use Policy, 46, pp. 163-177. Recuperado de http:// www.sciencedirect.com/science/article/pii/S0264837715000319

Khanna, M., Kochhar, N. y Palaniswamy, N. (2015). A Retrospective Impact Evaluation of the Tamil Nadu Empowerment and Poverty Alleviation (Pudhu Vaazhvu) Project. The Journal of Development Studies, 51(9), pp. 1210-1223. doi: http://www.tandfonline.com/doi/full/10.1080/00220388.2015.1028538

Marshall, G. R. (2009). Polycentricity, Reciprocity, and Farmer Adoption of Conservation Practices under Community-based Governance. Ecological Economics, 68(5), pp. 1507-1520. Recuperado de http://www.sciencedirect.com/science/ article/pii/S0921800908004722 
Meertens, D. (2004). Género, desplazamiento, derechos. En Bello, M. (ed.), Desplazamiento forzado. Dinámicas de guerra, exclusión y desarraigo, pp. 197-204. Bogotá: Universidad Nacional de Colombia.

Mettepenningen, E., Beckmann, V. y Eggers, J. (2011). Public Transaction Costs of Agri-environmental Schemes and their Determinants-Analysing Stakeholders' Involvement and Perceptions. Ecological Economics, 70(4), pp. 641650. Recuperado de http://www.sciencedirect.com/science/article/pii/ S0921800910004258

Mingol, I. (2007). La ética del cuidado: contribuciones a una transformación pacífica de los conflictos. Feminismo/s, 9, pp. 93-106. Recuperado de http://rua. ua.es/dspace/handle/10045/3643

Misión de Observación Electoral (MOE). (s. f.). Monografia politica electoral, departamento de Cundinamarca 1997 a 2007. Recuperado de http://moe.org.co/ home/doc/moe_mre/CD/PDF/cundinamarca.pdf

Naldi, L., Nilsson, P., Westlund, H. y Wixe, S. (2015). What is Smart Rural Development? Journal of Rural Studies 40, pp. 90-101. Recuperado de https://www. researchgate.net/publication/279634589_What_is_smart_rural_development

Paré, L. y Fuentes, T. (2007). Gobernanza ambiental y políticas públicas en áreas naturales protegidas: lecciones desde Los Tuxtlas. Recuperado de https://sendas99. files.wordpress.com/2009/05/gobernanzapareyfuentes.pdf

Parra-Peña, S., Isidro, R., Ordoñez, A., Acosta, C. (2013). Pobreza, brechas y ruralidad en Colombia. Coyuntura Económica, 43(1), pp. 15-36. Recuperado de http://www.repository.fedesarrollo.org.co/handle/11445/260

Pérez, B. (2006). Los grupos paramilitares en Bogotá y Cundinamarca, 1997 y 2005. Desafios, 14, pp. 338-381. Recuperado de http://www.redalyc.org/articulo.oa?id=359633159008

Pérez, F. (2005). Notas para un balance de las dinámicas de guerra y desplazamiento forzado en la región Central. En Redif, Acnur y Corporación Región (eds.), El desplazamiento en Colombia, pp. 101-123. Recuperado de http://www. catedras-bogota.unal.edu.co/web/ancizar/2007I/documentos/DesplazamientoColombia.pdf

Perry, S. (2010). La pobreza rural en Colombia. Recuperado de http://www.rimisp. org/wp-content/files_mf/1366386291DocumentoDiagnosticoColombia.pdf

Portolés, C. (2007). De la reclamación de la paz a la participación en las negociaciones: el feminismo pacifista. Feminismo/s, 9, pp. 15-30. Recuperado de http:// rua.ua.es/dspace/bitstream/10045/27218/1/Feminismos_9.pdf

Prager, K. (2010). Local and Regional Partnerships in Natural Resource Management: The Challenge of Bridging Institutional Levels. Environmental 
Management, 46(5), pp. 711-724. Recuperado de http://link.springer.com/ article/10.1007/s00267-010-9560-9

Programa de las Naciones Unidas para el Desarrollo [PNUD]. (2012). Cundinamarca frente a los objetivos del milenio. Estado de avance. Recuperado de http://www. pnud.org.co/sitio.shtml?apc=jAa-2--Ex=67110

Rahman, A. y Fals Borda, O. (1989). La situación actual y las perspectivas de la IAP en el mundo. Recuperado de http://www.gumilla.org/biblioteca/bases/ biblo/texto/COM199694_14-20.pdf

Ramírez-Miranda, C. (2014). Critical Reflections on the New Rurality and the Rural Territorial Development Approaches in Latin America. Agronomía Colombiana, 32(1), pp. 122-129. Recuperado de http://www.scielo.org.co/pdf/ agc/v32n1/v32n1a16.pdf

Rico, M. (1998). Género, medio ambiente y sustentabilidad del desarrollo. Recuperado de http://repositorio.cepal.org/bitstream/handle/11362/5867/S9800082_ es.pdf?sequence $=1$

Rodríguez-Becerra, M., Espinoza, G. y Wilk, D. (2002). Gestión ambiental en América Latina y el Caribe: evolución, tendencias y principales prácticas. Recuperado de http://documentacion.ideam.gov.co/openbiblio/bvirtual/019857/ GestionambientalenA.L.yelC/GestionAmb..pdf

Romero, G. (2012). Voices Around Us: Memory and Community Empowerment in Reconstruction Efforts in Colombia. International Journal of Transitional Justice, 6(3), pp. 547-557. Recuperado de http://ijtj.oxfordjournals.org/content/ early/2012/09/04/ijti.ij018

Ruiz, E. y Aldás, E. (2007). Género, conflicto y construcción de la paz: reflexiones y propuestas. Recuperado de http://m.web.ua.es/es/cem/publicaciones/feminismos/ numerospublicados/feminismo-s-9-genero-conflicto-y-construccion-de-la-pazreflexiones-y-propuestas.html

Sánchez-Muros, S. y Jiménez, M. L. (2013). Mujeres rurales y participación social: análisis del asociacionismo femenino en la provincia de Granada (España). Cuadernos de Desarrollo Rural, 10(72), pp. 223-242. Recuperado de http:// www.scielo.org.co/pdf/cudr/v10n72/v10n72a12.pdf

Shmite, S. (2009). Las mujeres rurales y su participación en los escenarios productivos actuales. La aljaba, 13 (13), pp. 117-131. Recuperado de http://www. scielo.org.ar/scielo.php?script=sci_arttext\&pid=S1669-57042009000100007

Taylor, B. y Van Grieken, M. (2015). Local Institutions and Farmer Participation in Agri-environmental Schemes. Journal of Rural Studies, 37, pp. 10-19. doi: http:// dx.doi.org/10.1016/j.jrurstud.2014.11.011 
Vicepresidencia de la República de Colombia. (2001). Panorama actual de Cundinamarca. Recuperado de http://historico.derechoshumanos.gov.co/Observatorio/Publicaciones/documents/2010/Estu_Regionales/04_03_regiones/ cundinamarca/cundinamarca.pdf

Villa, A. y Bracamonte, Á. (2013). Procesos de aprendizaje y modernización productiva en el agro del noroeste de México: los casos de la agricultura comercial de la costa de Hermosillo, Sonora y la agricultura orgánica de la zona sur de Baja California Sur. Estudios fronterizos, 14 (27), pp. 217-254. Recuperado de http://ref.uabc.mx/ojs/index.php/ref/article/view/82 